Taxonomía y sistemática

\title{
Peces de la Reserva de la Biosfera Selva El Ocote y presa Nezahualcóyotl (Malpaso) Chiapas, México
}

\author{
Fishes of the Biosphere Reserve Selva El Ocote and Nezahualcóyotl (Malpaso) reservoir, Chiapas, \\ Mexico
}

\author{
Manuel de Jesús Anzueto-Calvo*, Ernesto Velázquez-Velázquez y Adán Enrique Gómez-González \\ Museo de Zoología, Facultad de Ciencias Biológicas, Universidad de Ciencias y Artes de Chiapas, Libramiento Norte Poniente Núm. 1150, Col. Lajas Maciel, \\ 29039 Tuxtla Gutiérrez, Chiapas, México
}

Recibido el 4 de marzo de 2015; aceptado el 11 de abril de 2016

Disponible en Internet el 18 de agosto de 2016

\begin{abstract}
Resumen
Se presenta el inventario de peces de la Reserva de la Biosfera Selva El Ocote y presa Nezahualcóyotl (Malpaso), incluyendo el río Totopac en Chiapas, México. El material se recolectó en 14 muestreos realizados en 83 puntos entre marzo de 2007 y septiembre de 2009; además, se incluyen 4 campañas adicionales de 2014; en total, se recolectaron 22,034 ejemplares. El elenco sistemático está representado por 47 especies contenidas en 30 géneros, 16 familias y 10 órdenes. Las familias más representativas fueron Cichlidae y Poeciliidae con 16 y 11 especies, respectivamente (57\% de las especies recolectadas). Seis especies están bajo alguna categoría de riesgo según la NOM-059-SEMARNAT-2010, la Lista Roja de la Unión Internacional para la Conservación de la Naturaleza y la American Fisheries Society: Potamarius nelsoni, Rhamdia guatemalensis, Rhamdia laluchensis, Priapella intermedia, Xiphophorus clemenciae y Paraneetroplus hartwegi. Cuatro especies son exóticas: Oreochromis mossambicus, Oreochromis niloticus, Parachromis managuensis y Tilapia zillii.

Derechos Reservados (C) 2016 Universidad Nacional Autónoma de México, Instituto de Biología. Este es un artículo de acceso abierto distribuido bajo los términos de la Licencia Creative Commons CC BY-NC-ND 4.0.
\end{abstract}

Palabras clave: Ictiofauna; Riqueza; El Ocote; Presa Nezahualcóyotl

\begin{abstract}
An inventory of the fishes of the Selva El Ocote Biosphere Reserve and Nezahualcóyotl (Malpaso) dam, including the Totopac River in Chiapas, Mexico, is presented. The material was collected from 14 sampling campaigns in 83 points between March 2007 and September 2009 . Four more samplings were performed in 2014. In total 22,034 specimens were collected, the systematic list of the fishes comprises 47 species contained in 30 genera, 16 families and 10 orders. The most representative families were Cichlidae and Poeciliidae with 16 and 11 species respectively (57\% of collected individuals). Six species are under some risk category according to NOM-059-SEMARNAT-2010, the IUCN Red List and the American Fisheries Society: Potamarius nelsoni, Rhamdia guatemalensis, Rhamdia laluchensis, Priapella intermedia, Xiphophorus clemenciae, and Paraneetroplus hartwegi. Four species are exotic: Oreochromis mossambicus, Oreochromis niloticus, Parachromis managuensis, and Tilapia zillii.

All Rights Reserved (C 2016 Universidad Nacional Autónoma de México, Instituto de Biología. This is an open access item distributed under the Creative Commons CC License BY-NC-ND 4.0.
\end{abstract}

Keywords: Ichthyofauna; Richness; El Ocote; Nezahualcóyotl dam

\footnotetext{
* Autor para correspondencia.

Correo electrónico: manuel.anzueto@unicach.mx (M.d.J. Anzueto-Calvo). La revisión por pares es responsabilidad de la Universidad Nacional Autónoma de México.
}

\section{Introducción}

La Reserva de la Biosfera Selva el Ocote (REBISO), localizada al este del estado de Chiapas, México, constituye un área de gran interés biológico para la conservación, ya que forma parte 
de las regiones hidrológicas prioritarias (Malpaso-Pichucalco) y terrestres (selva Zoque-La Sepultura) del país establecidas por la Comisión Nacional Para el Uso y Conocimiento de la Biodiversidad (Arriaga-Cabrera, Aguilar y Alcocer, 2002).

La REBISO y la presa Malpaso forman parte de la cuenca hidrológica media del Grijalva, una de las cuencas más importantes de México ya que es la segunda más caudalosa del país, con un área aproximada de $58,000 \mathrm{~km}^{2}$ (González-Villareal, 2009), además, junto con la cuenca del río Usumacinta poseen una riqueza ictiofaunística conformada por 150 especies (INE, 2007) y particularmente para la parte media y alta del Grijalva, Maza-Cruz (2014) registra 53 especies. A lo largo del cauce del río Grijalva se han construido 4 grandes presas hidroeléctricas: la Angostura, Chicoasén, Malpaso y Peñitas (Gómez-González, Velázquez-Velázquez, Anzueto-Calvo y Maza-Cruz, 2015), las mismas que han modificado de manera importante el hábitat acuático al convertirlo de un ambiente lótico a uno léntico. Estas modificaciones trajeron consigo la fragmentación del hábitat que ha impedido el movimiento de varias especies periféricas hacia las aguas interiores de la cuenca del Grijalva, lo cual permitió que los nuevos ambientes creados fueran objeto de numerosas introducciones de especies invasoras (ej. las diferentes especies de tilapias y carpas) que han mermado las poblaciones de las especies nativas (Velázquez-Velázquez, Contreras-Balderas, Domínguez-Cisneros y Gómez-González, 2013).

\section{Materiales y métodos}

La REBISO y la presa Malpaso se localizan hacia la porción occidental del territorio chiapaneco ( $16^{\circ} 45^{\prime} 42^{\prime \prime}$ - $17^{\circ} 13^{\prime} 42^{\prime \prime}$ N, 93 54 '19” - 93²1'20” O), ubicadas en los municipios de Ocozocoautla de Espinosa, Cintalapa de Figueroa, Tecpatán de Mezcalapa y Jiquipilas, Chiapas (Moreno-Moreno, 1995; INESemarnat, 2000).

Para documentar la riqueza de especies se realizaron 14 campañas de muestreo en el período comprendido de marzo de 2007 a septiembre 2009, en el cual se establecieron 83 estaciones de muestreo (fig. 1). Las localidades se eligieron para cubrir diferentes tipos de hábitat dentro del polígono de la REBISO y sus zonas de influencia: rápidos y remansos en aguas superficiales y subterráneas, así como en el embalse de la presa Malpaso y sus tributarios, las mismas que se georreferenciaron utilizando un GPS Sport-TrakMap, marca Magellan.

La recolecta de ejemplares se realizó por localidad en cada uno de los recorridos. Se utilizó un equipo de electro pesca Smith Root 12A back pack, en transectos de 100 m; atarraya tipo camaronera de $6 \mathrm{~m}$ de diámetro y 0.5 pulgadas de luz de malla, 10 lances en cada sitio de muestreo; red agallera de $20 \mathrm{~m}$ de largo $\times 2 \mathrm{~m}$ de altura con luz de malla de 2.25 pulgadas, colocadas por $2 \mathrm{~h}$; y red tipo chinchorro de $3 \mathrm{~m}$ de largo $\times 2 \mathrm{~m}$ de altura y luz de malla de $5 \mathrm{~mm}$ por $30 \mathrm{~min}$.

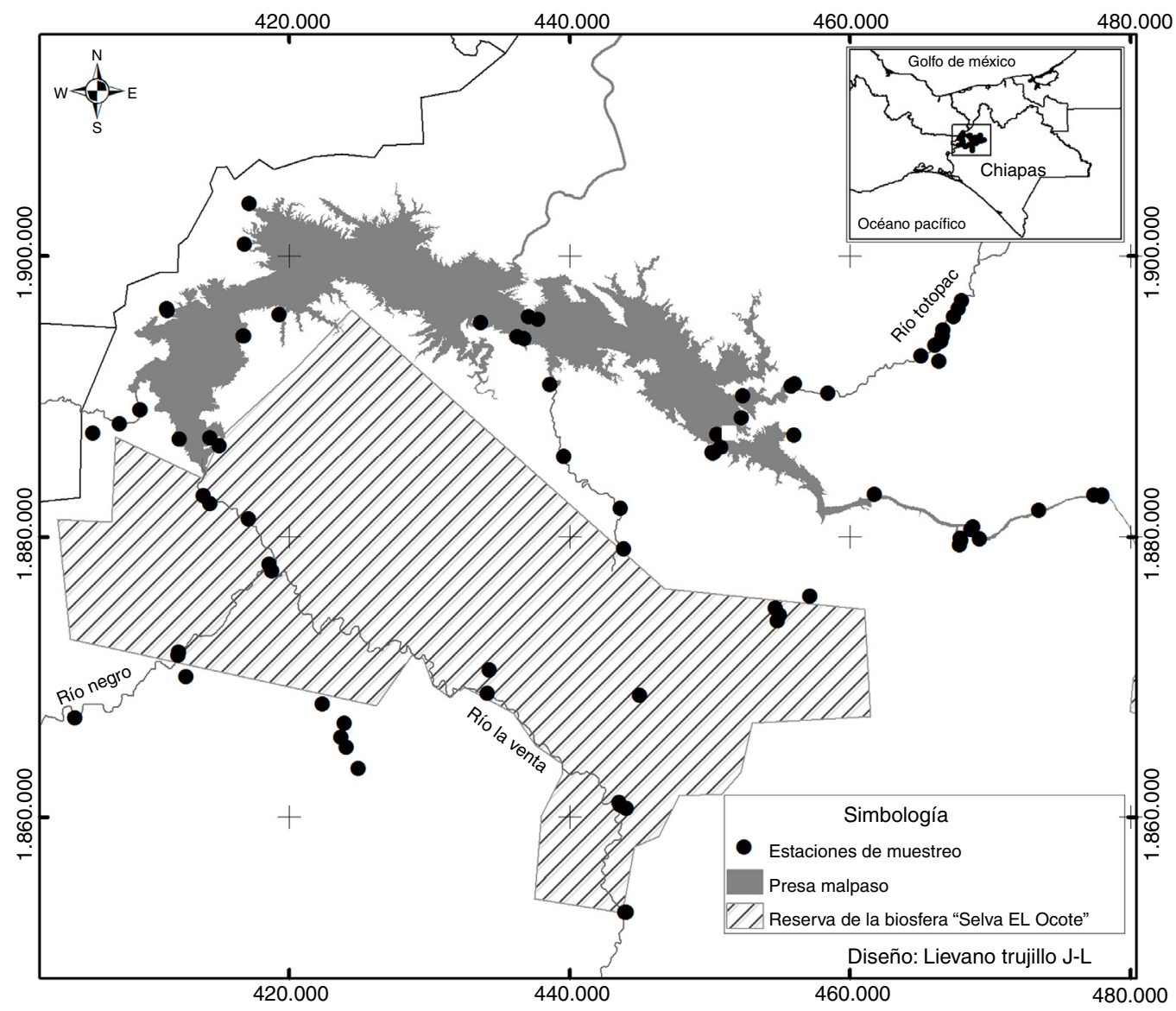

Figura 1. Estaciones de muestreo en la REBISO y presa Malpaso, Chiapas, México. 
La identificación taxonómica de los peces se realizó utilizando literatura básica y claves (Álvarez-del Villar, 1970; Castro-Aguirre, Espinoza-Pérez y Schmitter-Soto, 1999; Miller, Minckley y Norris, 2005; Velasco-Colín, 1976), así como descripciones originales y revisiones sistemáticas recientes. Los ejemplares capturados de cada especie fueron fotografiados, etiquetados y fijados en formalina al 10\%, de acuerdo con el método de Lagler, Bardach, Miller y May-Pasino (1984). Posteriormente, se trasladaron a la Colección de Peces de la Facultad de Ciencias Biológicas de la Universidad de Ciencias y Artes de Chiapas (MZ-P-UNICACH), donde se etiquetaron nuevamente y se colocaron en frascos de polietileno, conteniendo alcohol al $70 \%$ (anexo).

El arreglo taxonómico de las especies se realizó con base en el criterio de Nelson (2006) para el nivel de orden y familia; la ortografía y reconocimiento de autor y año de cada especie se realizaron de acuerdo a la revisión en línea del catálogo de Eschmeyer (2013), excepto en Rhamdia guatemalensis, para la cual se tomaron en cuenta los criterios de Weber y Wilkens (1998) y Perdices, Bermingham, Montilla y Doadrio (2002). Con el propósito de conocer la tolerancia histórica a la salinidad de las especies, estas se clasificaron considerando la categoría ecológica de Myers (1938). Adicionalmente se consultó la Colección Ictiológica de ECOSUR, Unidad San Cristóbal, para la revisión de los registros disponibles de la zona de estudio.

\section{Resultados}

Se recolectó un total de 22,034 ejemplares pertenecientes a 10 órdenes, 16 familias y 30 géneros, los mismos que conformaron el inventario con un total de 47 especies. Las familias Cichlidae y Poeciliidae fueron las mejores representadas con 15 y 10 especies, respectivamente (55\% de las especies recolectadas), mientras que las menos representadas fueron las familias Ictaluridae, Atherinopsidae, Synbranchidae, Gerreidae y Sciaenidae, en las cuales solo se registró una especie. Seis especies de peces están bajo alguna categoría de riesgo según la NOM059-SEMARNAT-2010 (Semarnat, 2010), la Lista Roja de la IUCN (IUCN, 2014) y la AFS (Jelks et al., 2008); Potamarius nelsoni, R. guatemalensis, Rhamdia laluchensis, Priapella intermedia, Xiphophorus clemenciae y Paraneetroplus hart$w e g i$. De las 5 especies anteriores, 3 son endémicas de la cuenca; $P$. nelsoni, X. clemenciae y $P$. hartwegi, además 4 especies son exóticas: Oreochromis mossambicus, Oreochromis niloticus, Parachromis managuensis y Tilapia zillii. Basadas en su afinidad ecológica 8 corresponden al componente dulceacuícola primario, 29 al dulceacuícola secundario y 10 al conjunto periférico (vicario).

Listado de las especies de peces de la REBISO y presa Malpaso, Chiapas.* Especie bajo alguna categoría de riesgo $(\mathrm{Pr}=$ sujeta a protección especial, $\mathrm{A}=$ amenazada) de acuerdo a la NOM-059-SEMARNAT-2010, a la UICN ( $\mathrm{LC}=$ preocupación menor, $\mathrm{VU}=$ vulnerable) y a la AFS ( $\mathrm{T}=$ amenazada), **especie exótica, CE: categoría ecológica $(\mathrm{Pe}=$ periférica, $\mathrm{DP}=$ dulceacuícola primaria, $\mathrm{DS}=$ dulceacuícola secundaria).
Orden Clupeiformes

I. I Familia Clupeidae

1. Dorosoma anale Meek $1904 \mathrm{Pe}$

2. Dorosoma petenense (Günther 1867) Pe Orden Cypriniformes

II. Familia Catostomidae

3. Ictiobus meridionalis (Günther 1868) DP Orden Characiformes

III. Familia Characidae

4. Astyanax aeneus (Günther 1860) DP

5. Brycon guatemalensis Regan 1908 DP Orden Siluriformes

IV. Familia Ictaluridae

6. Ictalurus meridionalis (Günther 1864) DP

V. Familia Ariidae

7. Cathorops kailolae Marceniuk y Betancur-R. $2008 \mathrm{Pe}$

8. Potamarius nelsoni (Evermann y Goldsborough 1902) $\mathrm{Pe} * \mathrm{Pr}$

VI. Familia Heptapteridae

9. Rhamdia guatemalensis (Günther 1864) DP * Pr

10. Rhamdia laluchensis Weber, Allegrucci y Sbordoni $2003 \mathrm{DP} * \mathrm{~T}$

11. Rhamdia laticauda (Kner 1858) DP

Orden Batrachoidiformes

VII. Familia Batrachoididae

12. Batrachoides goldmani Evermann y Goldsborough $1902 \mathrm{Pe}$

Orden Atheriniformes

VIII. Familia Atherinopsidae

13. Atherinella alvarezi (Díaz-Pardo 1972) Pe

Orden Beloniformes

IX. Familia Belonidae

14. Strongylura hubbsi Collette $1974 \mathrm{Pe}$

X. Familia Hemiramphidae

15. Hyporhamphus mexicanus Álvarez $1959 \mathrm{Pe}$

Orden Cyprinodontiformes

XI. Familia Profundulidae

16. Profundulus labialis (Günther 1866) DS

17. Profundulus punctatus (Günther 1866) DS

XII. Familia Poeciliidae

18. Gambusia yucatana Regan 1914 DS

19. Gambusia sexradiata Hubbs 1936. DS

20. Heterandria bimaculata (Heckel 1848) DS

21. Poecilia mexicana Steindachner 1863 DS

22. Poecilia sphenops Valenciennes 1846 DS

23. Poeciliopsis fasciata (Meek 1904) DS

24. Poeciliopsis hnilickai Meyer y Vogel 1981 DS

25. Poeciliopsis pleurospilus (Günther 1866) DS

26. Priapella intermedia Álvarez y Carranza 1952 DS * Pr LC

27. Xiphophorus clemenciae Álvarez 1959 DS * A

28. Xiphophorus hellerii Heckel 1848 DS

Orden Synbranchiformes

XIII. Familia Synbranchidae

29. Ophisternon aenigmaticum Rosen y Greenwood 1976 DP 
Orden Perciformes

XIV. Familia Gerreidae

30. Eugerres mexicanus (Steindachner 1863) Pe

XV. Familia Sciaenidae

31. Aplodinotus grunniens Rafinesque $1819 \mathrm{Pe}$

XVI. Familia Cichlidae

32. Amphilophus macracanthus (Günther 1864) DS

33. Cichlasoma salvini (Günther 1862) DS

34. Cichlasoma trimaculatum (Günther 1867) DS

35. Cichlasoma urophthalmus (Günther 1862) DS

36. Oreochromis mossambicus (Peters 1852) DS **

37. Oreochromis niloticus (Linnaeus 1758) DS **

38. Parachromis managuensis (Günther 1867) DS **

39. Paraneetroplus bifasciatus (Steindachner 1864) DS

40. Paraneetroplus hartwegi (Taylor y Miller 1980) DS * A VU

41. Paraneetroplus regani (Miller 1974) DS

42. Paraneetroplus melanurus (Günther 1862) DS

43. Paraneetroplus cf. zonatus DS

44. Petenia splendida Günther 1862 DS

45. Theraps pearsei (Hubbs 1936) DS

46. Thorichthys helleri (Steindachner 1864) DS

47. Tilapia zillii (Gervais 1848) DS **

\section{Discusión}

De las 92 especies continentales que Gómez-González et al. (2015) reportan para la cuenca del río Grijalva, 47 se documentaron en la REBISO y presa Malpaso, lo que corresponde a $51 \%$ de la ictiofauna de la cuenca. Más de la mitad (57\%) de las especies recolectadas se concentraron en 2 familias (Cichlidae y Poeciliidae), estos resultados son similares a lo descrito por Myers (1966) y Matamoros, McMahan, Chakrabarty, Albert y Schaefer (2015) quienes mencionan que estos grupos representan más de la mitad de la ictiofauna de Centroamérica y corroborado por Rodiles-Hernández, González-Díaz y Chan-Sala (2005), Espinosa-Pérez, Valencia-Díaz y Rodiles-Hernández (2011) y Velázquez-Velázquez et al. (2013) quienes reportan más del 30\% de la ictiofauna del estado de Chiapas. De las especies endémicas, $P$. hartwegi es la más ampliamente distribuida en 17 de los 83 puntos de muestreo, mientras que la menos distribuida es $X$. clemenciae en 4 puntos de muestreo. La especie $R$. laluchensis es un pez ciego que solo habita en la cueva de la localidad de la Lucha, municipio de Ocozocoautla de Espinosa, Chiapas y Santa María Chimalapa, Oaxaca (Weber, Allegrucci y Sbordoni, 2003), sin embargo no es considerada en ninguna categoría de la NOM-059-SEMARNAT-2010.

Espinosa-Pérez, Fuentes-Mata y Gaspar-Dillanes (1993) y Miller et al. (2005), documentaron la distribución de Cichlasoma trimaculatum y Amphilopus macracanthus en la vertiente del Pacífico, sin embargo, en este trabajo al igual que en el de González, Quiñones, Velásquez y Rodiles-Hernández (2008), ambas especies se recolectaron en la REBISO y en la presa Malpaso. Miller et al. (2005) mencionan que en 1998 se capturaron dos ejemplares de A. macracanthus para la zona de la REBISO y atribuyen su presencia en esta zona debido a la introducción para acuicultura, sin embargo, en este trabajo y en el de González et al. (2008) se apoya la teoría de que esta región pudo haber conectado a las 2 vertientes (Atlántico y Pacífico) considerando los cambios que pudieron ocurrir en el Plioceno, producto de la intensa actividad tectónica y volcánica (Johnson, 1989; Maldonado-Koerdell, 1964).

Las únicas especies que aparecen consideradas por la UICN son: $P$. intermedia y $P$. hartwegi enlistadas en preocupación menor y vulnerable, respectivamente. $P$. nelsoni, $R$. guatemlensis y $P$. intermedia aparecen en la NOM-059SEMARNAT-2010 como sujetas a protección especial. $X$. clemenciae, está considerada como amenazada de acuerdo a la NOM-059-SEMARNAT-2010 y a la AFS, la cual se adiciona al grupo de especies junto a Priapella intermedia y Paraneetroplus regani, endémicas de la cuenca del río Coatzacoalcos (Contreras-Balderas, Almada-Villela, Lozano-Vilano y GarcíaRamírez, 2003). En este trabajo se registra a $X$. clemenciae para la REBISO en la cuenca del Grijalva; su presencia en esta cuenca podría servir de base para reconsiderar su estatus y asegurar su supervivencia, que es comúnmente confundida con Xiphophorus helleri debido a semejanzas morfológicas (Gómez-González, Velázquez-Velázquez y Anzueto-Calvo, 2014) pero distinta al estudiarlas a detalle, además su registro corresponde a 8 localidades del área de estudio en arroyos y ríos que drenan a la presa Malpaso.

La regionalización propuesta por Lozano-Vilano y ContrerasBalderas (1987) para Chiapas en subprovincias ictiogeográficas incluye a la zona de la REBISO y la presa Nezahualcóyotl dentro de la subprovincia I (Sierra Atravesada o Tehuantepeca), considerándola con fauna poco distintiva tomando como ejemplo el río Cintalapa con tan solo 6 especies, lo cual no coincide con los resultados de este estudio y hace evidente los pocos trabajos realizados en esta zona.

El $62 \%$ de las especies del área de estudio pertenecen al grupo dulceacuícola secundario principalmente por que 27 especies se agrupan en las 2 familias más representativas, Cichlidae y Poeciliidae, las mismas que son dominantes en la mayoría de los sistemas fluviales de América Central (Miller, 1966; Myers, 1966). Estos resultados son similares a lo que Gómez-González et al. (2015) reportan para la cuenca del río Grijalva.

Se ha confirmado la presencia de al menos 4 especies exóticas pertenecientes a la familia Cichlidae: $O$. niloticus, O. mossambicus y $T$. zillii, todas originarias de África y $P$. managuensis procedente de Centroamérica, introducidas con fines de pesca y acuicultura en el embalse de la presa Nezahualcóyotl (Malpaso). El registro de O. niloticus para el área de estudio es desde 1995 (Moreno-Moreno, 1995), mientras que a $P$. managuensis lo documentan por primera vez González et al. (2008). La especie con mayor distribución es $P$. managuensis en 20 puntos de muestreo. Es necesario realizar una monitorización de las especies exóticas, ya que se desconoce el impacto que puedan ocasionar sobre la biota nativa, así como en las pesquerías basadas en especies nativas de gran valor comercial, como la tenguayaca (Petenia splendida) y el bagre (Ictalurus meridionalis). Cabe señalar que no se capturó ninguna de las especies de carpas distribuidas en el estado de Chiapas. 
A pesar del notable avance en el conocimiento de la diversidad de peces de la REBISO y la presa Malpaso, es necesario continuar con investigaciones de diversa índole. Entre ellas está el esclarecer el estatus taxonómico de algunas especies, principalmente las del género Paraneetroplus, ya que la gran similitud morfológica entre las especies dificulta su identificación.

\section{Agradecimientos}

Expresamos nuestro sincero agradecimiento a la Comisión Nacional de Áreas Naturales Protegidas, en especial a los directivos de la Reserva El Ocote, por su apoyo en la logística y a la Comisión Nacional para el Conocimiento y Uso de la Biodiversidad, por su contribución en el financiamiento para la realización de este trabajo. Al Dr. Wilfredo Matamoros por sus contribuciones a este trabajo, al Biól. Ever U. Vázquez Pérez, por su apoyo en el campo y al Biól. Jorge Luis Liévano Trujillo por la elaboración de la figura.

\section{Anexo. Números de catálogo de peces ingresados en la colección ictiológica (MZ-P-UNICACH)}

Amphilophus macracanthus (Günther 1864): 1653, 3968, 4007, 1423, 1438, 1568, 1580, 1584, 2315, 2324, 2703, 2712, 3195, 3694, 1459, 1600, 1606, 2970, 3952, 2980, 1893, 1920. Aplodinotus grunniens - Rafinesque,1819: 1507, 2540, 2089, 2699, 2916, 2928, 1886, 1723, 2929, 2017, 2564, 2241, 1378, 4056. Astyanax aeneus (Günther, 1860): 1347, 1544, 1667, 1763, 1950, 1977, 2164, 2546, 2787, 2907, 3781, 3837, 3869, 3989, 4647, 2826, 3593, 3621, 3958, 4000, 1518, 1687, 1967, 1995, 2915, 3511, 3738, 3806, 3846, 3754, 3860, 4061, 4137, 1855, 2081, 2086, 2251, 3562, 3598, 3994, 1743, 2956, 3575, 3585, $3961,2014,1272,1363,1425,1575,1577,1582,1613,1822$, 2113, 2119, 2135, 2316, 2319, 2702, 2708, 2717, 3190, 3196, 3691, 2237, 2245, 2234, 2595, 2598, 1645, 2049, 2223, 2227, 2575, 2577, 1469, 1610, 1946, 2097, 2099, 2343, 2346, 2347, 2255, 1516, 2738, 2747, 3662, 3687, 1817, 2278, 2275, 1261, 1262, 1314, 1309, 1396, 1397, 1441, 1446, 2798, 2806, 2033, 2207, 2213, 1400, 1449, 1451, 3710, 3761, 3773, 4124, 4130, 1344, 1376, 1419, 1484, 1558, 1799, 1832, 2145, 2326, 2751, $3630,3633,2126,2131,2282,2310,3156,3159,3666,1728$, $1755,2129,2305,2735,3161,3164,3638,3669,3678,2104$, 2107, 1547, 1550, 1618, 1623, 1656, 2952, 1636, 1703, 1912, 2108, 2284, 2285, 2297, 2750, 2760, 3168, 3170, 3176, 3645, $3648,3824,3840,3944,1766,3682,2726,3655,3676,1263$, 1266, 1304, 1503, 1668, 1989, 2177, 2509, 2532, 2556, 2793, 3515, 3742, 3801, 3896, 4042, 4102, 4144, 5016, 5041, 5042, 5053, 4119, 1529, 1734, 2551, 1257, 1293, 1393, 1601, 1608, 1934, 2142, 2288, 2294, 3184, 3187, 3635, 3640, 3642, 1880, 1908, 2060, 2066, 2811, 2977, 3579, 3982, 4050, 1276, 1280, 1282, 1294, 1296, 1365, 1411, 1866, 1895, 1903, 2079, 2087, 2260. Atherinella alvarezi (Díaz-Pardo, 1972): 1496, 1513, 1658, 1802, 1980, 2160, 2523, 2782, 2879, 3785, 3938, 3872, 3992, 2985, 3003, 3805, 3931, 3757, 4059, 4113, 1759, 1771, 2249, 2815, 3563, 3967, 1424, 1439, 2314, 1915, 1918, 1537, $1712,1715,1846,1835,1844,2772,2860,3976,3765,3613$, 3886, 4024, 1993, 2009, 2011, 2176, 2510, 2557, 2933, 3727,
3733, 3811, 3880, 4044, 2869, 1540, 1541, 1910, 3610, 1278, 1643, 2959, 2962. Batrachoides goldmani Evermann y Goldsborough 1902: 2508, 4293. Brycon guatemalensis Regan 1908: 1342, 2169, 2174, 2919, 4006, 1974, 2007, 2696, 3930, 4067, 1897, 2046, 2845, 2954, 2998, 4087, 1565, 1590, 1841, 2117, 2122, 2714, 3191, 2583, 1463, 1466, 1598, 1911, 1945, 2100, 2336, 2339, 2780, 2884, 1736, 2025, 1356, 1445, 1349, 1798, 2733, 2745, 2972, 2111, 2296, 3171, 3174, 3637, 3652, 2055, 2724, 2728, 3673, 3686, 1319, 1680, 2558, 5033, 1811, 1815 , 2036, 2188, 2058, 2071, 2238, 2837, 2993, 3608, 3984, 4010, 4055, 1287, 1300, 1367, 1375, 1383, 1406, 1410, 1454, 1457, 1629, 1892, 2042, 2077, 2247, 1254. Cathorops kailolae Marceniuk y Betancur-R. 2008: 1510, 1508, 1669, 1685, 1805, 1952, 1963, 2172, 2515, 2903, 3834, 2820, 3590, 3955, 4014, 1686, $1786,1968,2695,2914,3506,3736,3847,3926,3861,4062$, 4134, 1884, 2842, 4088, 2781, 1709, 1727, 2206, 2229, 2769, 2857, 2924, 3497, 3714, 1796, 2198, 3949, 3997, 3941, 1673, 2797, 2932, 3519, 3744, 3800, 4046, 4100, 5031, 2555, 3721, 2021, 2204, 2562, 2567, 1741, 2994, 3983, 4052, 1286, 1340, 1369, 1373, 1414, 1630, 1642, 3625. Cichlasoma salvini (Günther 1862): 1327, 1939, 1497, 1803, 2152, 2531, 3299, 3707, 4070, 3004, 3592, 3623, 3971, 3957, 4004, 1671, 4066, 3555, 1857, 3599, 2221, 1747, 2900, 3711, 3763, 3775, 3857, 4129, $1872,1331,1333,1377,1559,1829,2323,3628,3617,3950$, 3178, 3829, 3841, 4023, 4077, 1927, 3609. Cichlasoma trimaculatum (Günther 1867): 1429, 1563, 3199, 1620, 2098, 2345, 1631, 5056, 5146, 1288. Cichlasoma urophthalmus (Günther 1862): 5056, 5146, 1288. Dorosoma anale Meek 1904: 1506, $1678,1682,1955,1961,2168,2173,2834,1676,1782,1966$, 2000, 2191, 2537, 2832, 2697, 2910, 2912, 2925, 3509, 3304, $3735,3753,1883,2045,2843,2995,4085,2052,2228,1528$, 1914, 2777, 2883, 3766, 1788, 2056, 1876, 2183, 1706, 2559, 2700, 2930, 3719, 3746, 3798, 4143, 1809, 2037, 2189, 2554, 2794, 2202, 2561, 2921, 2059, 2074, 2239, 2839, 2979, 2992, 3583, 3620, 3979, 4012, 4053, 1295, 1297, 1368, 1372, 1405, 1407, 1412, 1455, 1627, 1775, 1868, 1887, 1896, 1901, 2041, 2078, 2242, 2591, 2601, 2987, 2991, 4093. Dorosoma petenense (Günther 1867): 1650, 1499, 1532, 1697, 1765, 2030, 2167, 2526, 2542, 2790, 2849, 2902, 3703, 3836, 3822, 3934, 3859, 4646, 2824, 2973, 3591, 3624, 3970, 3959, 4003, 4015, $1511,1698,1973,2005,2535,2865,3510,3522,3740,3850$, 3928, 3752, 3863, 4060, 4140, 2966, 3557, 1856, 2080,2085, 2252, 2585, 2941, 2942, 3604, 2957, 3576, 3962, 2015, 2194, $1417,2050,2222,5055,2572,2581,1474,1523,1722,1725$, 1792, 1836, 2211, 2216, 2767, 2855, 3499, 3712, 3760, 3778, 2967, 3951, 3998, 3827, 3844, 3884, 3889, 4075, 1875, 1505, 1713, 1990, 2012, 2543, 2937, 3513, 3745, 3893, 4098, 4145, 4996, 5030, 5032, 5052, 2566, 2874, 2885, 3866, 4120, 1742, 2065, 2975, 3581, 3985, 4049, 1260, 1275, 1279, 1283, 1299, 1370, 1374, 1413, 1869, 1894, 1902, 2961. Eugerres mexicanus (Steindachner 1863): 2043, 1379, 1456, 1899, 4057. Gambusia sexradiata Hubbs 1936: 5173. Gambusia yucatana - Regan, 1914: 1720, 1838. Heterandria bimaculata (Heckel 1848): 2148, 2545, 2888, 2920, 3787, 2829, 2963, 1948, 2082, 2083, 2254, 2587, 2588, 2816, 2946, 2950, 3551, 3552, 4009, 2199, 2732, 2739, 2740, 2749, 3659, 1316, 1311, 1394, 1440, 2804, 2103, 2683, 3189, 3697, 2132, 2291, 2309, 3154, 3665, 
$1753,2302,2306,2742,2744,3163,3667,3670,2105,2299$, $1546,1622,1624,1731,2289,2831,3181,3695,2721,2727$, $2731,3656,3696,1258,1277,1391,1603,1621,1936,2140$, 2143, 2287, 2293, 3186, 3188, 3634, 2258, 2259. Hyporhamphus mexicanus Alvarez 1959: 1250, 1512, 1655, 1953, 1983, 2163, 2527, 3704, 3991, 1714, 2027, 2863, 2864, 3716, 3891, 1371, 1701, 2958, 2960, 3559. Ictalurus meridionalis (Günther 1864): 1500, 1675, 1962, 3588, 4017, 1972, 2002, 2193, 2536, 2698, 2917, 3507, 3303, 3741, 3851, 3929, 1885, 2047, 2846, 2997, 3616, 4089, 1359, 1430, 1436, 1852, 2115, 2123, 2704, 2705, 2713, 3198, 1944, 2093, 2102, 1717, 1740, 2026, 2034, 2212, 2768, 2774, 2852, 2859, 3826, 3845, 4076, 2057, 3685, $1873,1700,3715,3748,3879,4041,4103,1812,1813,2563$ 4118, 2999, 4084, 1404, 1453, 1773, 1776, 1889, 2038, 2076 , 4094. Ophisternon aenigmaticum Rosen y Greenwood 1976: 2341, 2276, 1420, 3157, 3165, 3679, 3653, 1303. Oreochromis mossambicus (Peters 1852): 1553, 2124, 1292. Oreochromis niloticus (Linnaeus 1758): 1492, 1564, 1519, 1696, 1979, 2158, 2165, 2514, 2522, 2512, 2784, 2791, 2878, 2895, 3298, $3833,3788,3820,3933,4072,2821,2982,3596,1437,2048$, 2205, 2226, 2091, 3731, 3770, 2971, 2186, 3881, 4043, 2064, 1907. Parachromis managuensis (Günther 1867): 1648, 1493, 1531, 1649, 1806, 1978, 1954, 1959, 2157, 2161, 2528, 2788, 2875, 2894, 2891, 2892, 2905, 3297, 3570, 3568, 3783, 4071, 2827, 3594, 3622, 4016, 1699, 3512, 3305, 3925, 3755, 4139, $1779,2817,2939,3561,3603,3587,1646,2576,2579,1749$, 2776, 2882, 1721, 1724, 2886, 2890, 2899, 3728, 3776, 3813, $3854,4125,2197,3607,4657,3842$, 3888, 3943, 3946, 4022, 4078, 3516, 3725, 3810, 3894, 2019, 2922, 4689, 4690, 2813, 2838, 2978, 3580, 4082, 1862, 1900. Paraneetroplus bifasciatus (Steindachner 1864): 1329, 1336, 1491, 1498, 1521, 1533, 1663, 1694, 1756, 1804, 1956, 1960, 2171, 2541, 1778, 1969, 2003, 2192, 1859, 1560, 1572, 1659, 1461, 1611, 1942, 2349, $1535,1718,1738,1849,2023,2031,1357,1452,1789,1839$, 1386, 1556, 1264, 1270, 1306, 1881, 2184, 1692, 1988, 1538, 2073, 1298, 1409, 1639, 1861, 1888, 1890. Paraneetroplus hartwegi (Taylor y Miller 1980): 3302, 3571, 3786, 3838, 3939, 3858, 4650, 3974, 4002, 2008, 3737, 3848, 4115, 4136, 3556, 3602, 3964, 3993, 1431, 1853, 3699, 3500, 3713, 3771, 3818, 4123, 3615, 3953, 2110, 3172, 3175, 3180, 3828, 4080, 3518, 3724, 3747, 3802, 3890, 4099, 4142, 3504, 3867. Paraneetroplus melanurus (Günther 1862): 1490, 1502, 1762, 3301, 3790, 4649, 2828, 2984, 1785, 3520, 2945, 3601, 1716, 1794, 2854, 3496, 3768, 2947, 1693, 2701, 5054, 2203, 3981, 4051, 1253. Paraneetroplus regani (Miller 1974): 1335, 1341, 2580, 1467, 1472, 1594, 1596, 1597, 1947, 2094, 2101, 2340, 1737, 2217, 2856, 2923, 1355, 1444, 1330, 1385, 1433, 1434, 1828, 2725, 2730, 3675, 3683, 1318, 3626. Paraneetroplus zonatus (Meek 1905): 1982, 2159, 2518, 2517, 2524, 2783, 2789, 2848, 2880, 2908, 2823, 2974, 1975, 2911, 2926, 3758, 2944, 2844, 2716, 2593, 1665, 2584, 2096, 2333, 2348, 1527, 2741, 3690, 1816, 2799, 2805, 1748, 1708, 1793, 1834, 2208, 2215, 2766, 2792, 2851, 1448, 2897, 3769, 3774, 1769, 2949, 3619, 1635, 2109, 2729, 3672, 3680, 2934, 2871, 2063, 1925, 1285. Petenia splendida Günther 1862: 1489, 1501, 1522, 1545, 1625, 1670, 1660, 1695, 1761, 1807, 1951, 1976, 2149, 2151, 2153, 2170, 2525, 2529, 2520, 2538, 2785, 2786, 2847, 2877, 2867, 2909, 2918,
$3295,3569,3566,3708,3779,3835,3819,3935,3937,3868$, 3986, 4068, 4146, 4645, 4651, 2088, 2590, 2825, 2835, 2983, 3005, 3589, 3972, 3956, 4005, 4018, 1689, 1783, 2001, 2534, 2694, 2913, 2927, 3508, 3306, 3734, 3809, 3932, 3756, 4063, 4111, 4141, 2965, 3554, 1757, 1772, 2084, 2814, 2940, 2943, 3564, 3597, 3965, 3995, 1898, 2996, 3577, 3586, 3960, 4008, 4086, 1644, 2053, 2220, 2571, 2578, 2256, 1517, 1746, 1913, 1916, 2549, 2778, 2881, 4081, 1536, 1711, 1729, 1735, 1797, 1848, 1833, 1845, 2024, 2028, 2210, 2214, 2230, 2770, 2850, 2861, 3495, 3501, 3975, 2896, 3709, 3730, 3764, 3772, 3817, 3855, 4127, 4132, 1791, 1826, 1850, 1871, 2196, 2948, 2951, 3612 , 3618, 3954, 3999, 3646, 3794, 3830, 3839, 3885, 3887, 3942, 4020, 4074, 1874, 2185, 1504, 1707, 1991, 2013, 2544, 2936, 3517, 3574, 3722, 3797, 3882, 3895, 4095, 1810, 2190, $2553,2795,2018,2200,2560,2565,2873,3505,3864,4116$, 1777, 1877, 2072, 2240, 2841, 2976, 3001, 3582, 1281, 1381, 1382, 1774, 1867, 1906, 2039, 2248, 2602, 2988, 2990, 3558, 3584, 4092, 1252. Poecilia mexicana Steindachner 1863: 1328, 1337, 1938, 1657, 1987, 2150, 2162, 2519, 2876, 2893, 2906, 3705,3782 , 3936, 3870, 3987, 4073, 2586, 2818, 1999, 3808, $3849,3924,4058,4112,4135,2232,2233,2830,2225,2569$, 1943, 2802, 2807, 2808, 1751, 1843, 2889, 2898, 3729, 3762, $3815,3856,4128,4133,1854,1870,1389,1421,1633,2322$, 2763, 3631, 2128, 2133, 2292, 2308, 3158, 3160, 1754, 2130, 2301, 2307, 2743, 2746, 3162, 3166, 3639, 3668, 2298, 1549, 1551, 1808, 3996, 1634, 1704, 1705, 1732, 1922, 1931, 2112, 2290, 2303, 2759, 2765, 3167, 3182, 3644, 3647, 3650, 3684, 1514, 2533, 2935, 3718, 3812, 3892, 4045, 4117, 1542, 1928, 1923, 2812, 2250, 2257. Poecilia sphenops Valenciennes 1846: 1495, 1997, 1320, 1364, 1416, 1573, 1578, 1589, 1614, 1937, 2114, 2120, 2138, 2317, 2328, 2707, 2710, 3192, 3197, 3692, 3701, 2224, 1462, 1464, 1470, 1595, 1599, 1607, 1919, 2095, 2332, 2337, 1477, 1481, 1825, 2265, 2269, 2280, 1353, 1403, 1554, 2719, 3658, 1265, 1269, 1308, 2181, 3502, 1602, 2061, 2067. Poeciliopsis fasciata (Meek 1904): 1494, 1652, 1986, 2166, 2521, 2547, 2868, 3784, 3940, 3873, 4069, 3006, 3969, 4019, 3927, 4065, 1758, 2589, 3560, 3963, 1274, 1324, 1362, $1426,1562,1566,1612,1840,2118,2136,2311,2320,2330$, 2711, 3194, 3702, 2573, 1460, 1471, 1588, 1604, 1941, 2334, 1478, 1483, 1585, 1818, 2272, 2264, 2270, 1917, 1543, 1719, 1847, 2029, 2209, 2773, 2862, 2887, 3977, 2901, 3732, 3816, $3853,4122,1343,1387,1418,1555,1830,2147,2327,2752$, 2761, 3606, 1548, 3614, 2757, 3177, 3649, 4021, 1767, 1515, 2010, 2175, 2938, 3723, 3883, 4048, 2872, 3503, 4121, 1726, 1641. Poeciliopsis hnilickai Meyer y Vogel 1981: 1480, 1593, 1820, 2271, 2267, 2274, 2279, 1733. Poeciliopsis pleurospilus (Günther 1866): 1998, 3807, 4114, 1271, 1326, 1358, 1422, 1570, 1576, 1583, 1616, 1851, 2116, 2137, 2321, 2329, 2706, 2718, 3193, 3201, 3698, 2570, 1473, 1475, 1609, 1619, 2090, 2335, 2342, 1476, 2125, 2737, 2748, 3663, 3688, 1479, 1591, $1819,2268,2263,2273,2277,1313,1310,1352,1401,1443$, $1447,1346,1637,1730,1932,2286,2758,3651,1768,2722$, 3674, 3681, 2720, 3654, 3677, 1256, 1267, 1307, 2182, 4047, 1878, 2062, 2069, 1865, 1905. Potamarius nelsoni (Evermann y Goldsborough 1902): 1487, 1509, 1691, 1958, 2539, 1784, 2833, 1882, 2779, 2231, 1790, 3945, 4091, 2187, 3799, 2035, 2840, 3000, 3980, 4054, 1380, 1626, 2040, 2075, 2243, 2600, 
2989. Priapella intermedia Alvarez y Carranza 1952: 1334, 2244, 2592, 2597, 2803, 1332, 1390, 1831, 2146, 2762, 3605, 3627, 3636. Profundulus labialis (Günther 1866): 1994, 1273, $1322,1360,1567,1581,1615,1824,2121,2139,2313,2325$, $2715,3200,3693,3700,2574,1458,1465,1468,1605,1940$, $2092,2338,2344,2736,3661,3689,1315,1395,1354,1402$, $1450,2127,2283,3155,3664,1752,2734,2106,2300,1552$, 1617, 1638, 1921, 1933, 2304, 2756, 3173, 3179, 2723, 1305, 1929. Profundulus punctatus (Günther 1866): 1321, 1427, 1569, 1801, 1823, 3183, 1482, 1586, 1821, 2262, 2266, 1399, 3657, 2552. Rhamdia guatemalensis (Quoy y Gaimard 1824): 1654, 1666, 1677, 1985, 2548, 3296, 3832, 3947, 3871, 3988, 2822, $1672,1781,1970,1996,3739,1325,1361,1428,1435,1561$, $1571,1579,1842,2134,2312,2318,2331,2709,1647,2582$, 2261, 1526, 3660, 1587, 2281, 1312, 1398, 1442, 4471, 2801, $1750,2032,2218,3814,4126,2754,3611,2755,3169,3643$, $3671,1255,1268,1317,1684,3514,3726,3804,4096,1879$, 1909, 1926, 4013, 1640, 1863. Rhamdia laluchensis Weber, Allegrucci y Sbordoni 2003: 1702, 2809, 2810. Rhamdia laticauda (Kner 1858): 1339, 1679, 1957, 1965, 2004, 2044, 4090, 2800, 2775, 4131, 2195, 1770, 2753, 1683, 2931, 1814, 2020, 2568, 2236, 2599. Strongylura hubbsi Collette 1974: 1486, 2154, 2530, 2511, 2955, 3578, 5017. Theraps pearsei (Hubbs 1936): 1520, 1534, 1651, 1681, 1764, 1984, 1964, 2155, 2516, 2796, 3300, 3567, 3706, 3789, 3821, 2836, 3973, 1780, 2006, $2866,3521,1858,3565,3600,3966,2054,1745,1739,2022$, 1787, 1837, 2953, 3793, 3843, 1690, 1992, 2180, 4097, 3720, 2016, 2201, 3865, 2070, 2981, 3002, 3978, 4011, 4083, 1284, 1366, 1408, 1628, 1891, 2986. Thorichthys helleri (Steindachner 1864): 1351, 1488, 1530, 1662, 1664, 1760, 1949, 1981, 2156, 2513, 2904, 3780, 3831, 3948, 3874, 3990, 4648, 2819, 3595, 4001, 1688, 1971, 3852, 3759, 3862, 4064, 4138, 3553, 1860, $1574,1674,2051,1744,2550,1710,2219,2771,2853,2858$, $3498,3767,3777,1345,1348,1384,1415,1485,1592,1800$, 1827, 3632, 2968, 3825, 4079, 2179, 3717, 3743, 3803, 4101, 2870, 1539, 1930, 2068, 1924, 1301, 1864. Tilapia zillii (Gervais 1848): 1661, 2969, 1904. Xiphophorus clemenciae Alvarez 1959: 2246, 4189, 2235, 2596, 2594, 2764, 3629, 1259, 1302, 1392, 1935, 2141, 2144, 2295, 3185, 3641, 2253. Xiphophorus hellerii Heckel 1848: 1338, 2964, 1350, 1388, 1432, 1557, 1632, 1795.

\section{Referencias}

Álvarez-del Villar, J. (1970). Peces mexicanos (Claves). México, D.F: Comisión Nacional Consultiva de Pesca.

Arriaga-Cabrera, L., Aguilar, V. y Alcocer, J. (2002). Aguas continentales y diversidad biológica de México. 91. Balancán. México, D.F: Conabio.

Castro-Aguirre, J. L., Espinoza-Pérez, H. S. y Schmitter-Soto, J. J. (1999). Ictiofauna estuarino-lagunar y vicaria de México. México, D.F: Editorial Limusa/Instituto Politécnico Nacional.

Contreras-Balderas, S., Almada-Villela, P., Lozano-Vilano, M. L. y GarcíaRamírez, M.E. (2003). Freshwater fish at risk or extint in Mexico, a checklist and review. Reviews in Fish Biology and Fisheries, 12, 241-251.

Eschmeyer, W.N. (Ed.), (2013). Genera, species, references. (consultado 1 Sep 2013). Disponible en: http://research.calacademy.org/research/ ichthyology/catalog/fishcatmain.asp.
Espinosa-Pérez, H., Fuentes-Mata, P. y Gaspar-Dillanes, M. T. (1993). Peces dulceacuícolas mexicanos. Listado Faunístico III. México, D.F: Instituto de Biología, UNAM.

Espinosa, H., Valencia-Díaz, X. y Rodiles-Hernández, R. (2011). Peces dulceacuícolas de Chiapas. En F. Álvarez (Ed.), Chiapas: estudios sobre su diversidad biológica (pp. 401-475). México, D.F: Instituto de Biología, Universidad Nacional Autónoma de México.

Gómez-González, A. E., Velázquez-Velázquez, E. y Anzueto-Calvo, M. J. (2014). Primer registro de Xiphophorus clemenciae (Cyprinodontiformes: Poeciliidae) en la cuenca del río Grijalva, México. Revista Mexicana de Biodiversidad, 85, 975-978.

Gómez-González, A. E., Velázquez-Velázquez, E., Anzueto-Calvo, M. J. y Maza-Cruz, M. F. (2015). Fishes of the Grijalva River basin of Mexico and Guatemala. Check List, 11, 1726.

González, D. A., Quiñones, R. M., Velásquez, M. J. y Rodiles-Hernández, R. (2008). Fishes of La Venta River in Chiapas, Mexico. Zootaxa, 1685, 47-54.

González-Villareal, F. J. (2009). Evaluación de la vulnerabilidad del sistema de presas del río Grijalva ante los impactos del cambio climático. México. D.F: Instituto Nacional de Ecología. Informe final, Clave: INE/A1-027/2009.

INE (Instituto Nacional de Ecología) (2007). La cuenca de los ríos Grijalva y Usumacinta. INE-Semarnat. (consultado 1 Sep 2014). Disponible en: http://www2.ine.gob.mx.

INE (Instituto Nacional de Ecología y Cambio Climático) /Semarnat (Secretaría de Medio Ambiente y Recursos Naturales). (2000). Programa de Manejo de la Reserva de La Biosfera Selva El Ocote. México. D.F: Instituto Nacional de Ecología, Semarnat.

IUCN (Red List of Threatened Species). (2014). La Lista Roja de Especies Amenazadas de la IUCN. Versión 2014-3. (consultado 1 Sep, 2014). Disponible en: www.iucnredlist.org.

Jelks, H. L., Walsh, S. J., Burkhead, N. M., Contreras-Balderas, S., Díaz-Pardo, E., Hendrickson, D. A., et al. (2008). Conservation status of imperiled North American freshwater and diadromous fishes. Fisheries, 33, 372-407.

Johnson, J. D. (1989). A biogeographic analysis of the herpetofauna of northwestern Nuclear Central America. Milwaukee Public Museum. Contributions in Biology and Geology, 76, 1-66.

Lagler, K. F., Bardach, J., Miller, R. R. y May-Pasino, D. R. (1984). Ictiología. México. D.F: AGT.

Lozano-Vilano, M. L. y Contreras-Balderas, S. (1987). Lista zoogeográfica y ecológica de la ictiofauna continental de Chiapas, México. The Southwestern Naturalist, 32, 223-236.

Maldonado-Koerdell, M. (1964). Geohistory and paleography of middle America. En R. Wauchope y R. C. West (Eds.), Handbook of middle American Indians (Vol. 1) (pp. 3-12). Austin: Natural Enviroment and Early Cultures. University Texas Press.

Matamoros, W. A., McMahan, C. D., Chakrabarty, P., James, A. S. y Schaefer, J. F. (2015). Derivation of the freshwater fish fauna of Central America. Cladistics, 31, 177-188.

Maza-Cruz, M. F. (2014). Riqueza y relación talla-peso de los peces del río Grijalva, Chiapas, México. Tesis. Tuxtla Gutiérrez, Chiapas: Universidad de Ciencias y Artes de Chiapas.

Miller, R. R. (1966). Geographical distribution of Central American freshwater fishes. Copeia, 4, 773-802.

Miller, R. R., Minckley, W. L. y Norris, S. M. (2005). Freshwater fishes of Mexico. Chicago: The University of Chicago Press.

Moreno-Moreno, R.A. (1995). Análisis de la actividad pesquera en la presa Nezahualcóyotl (Malpaso), Chiapas, México. Tesis de maestría. El Colegio de la Frontera Sur. San Cristóbal de Las Casas, Chiapas, México.

Myers, G. S. (1938). Fresh-water fishes and West Indian zoogeography. pp. 339-364. (1937) Smith Institute Annual Report.

Myers, G. S. (1966). Derivation of the freshwater fish fauna of Central America. Copeia, 4, 766-773.

Nelson, J. S. (2006). Fishes of the world (3. ${ }^{\text {a }}$ Ed.). Nueva York: John Wiley y Sons.

Perdices, A., Bermingham, E., Montilla, A. y Doadrio, I. (2002). Evolutionary history of the genus Rhamdia (Teleostei: Pimelodidae) in Central America. Molecular Phylogenetics and Evolution, 25, 172-189.

Rodiles-Hernández, R., González-Díaz, A. A. y Chan-Sala, C. (2005). Lista de peces continentales de Chiapas, México. Hidrobiológica, 15, 245-253. 
Semarnat (Secretaría de Medio Ambiente y Recursos Naturales). (2010). Norma Oficial Mexicana NOM-059-SEMARNAT-2010. Protección ambientalEspecies nativas de México de flora y fauna silvestres-Categorías de riesgo y especificaciones para su inclusión, exclusión o cambio-Lista de especies en riesgo. Diario Oficial de la Federación, 30 de diciembre, segunda sección: $1-77$.

Velasco-Colin, R. (1976). Los peces de agua dulce del Estado de Chiapas. Tuxtla Gutiérrez: Ediciones del Gobierno del Estado de Chiapas.

Velázquez-Velázquez, E., Contreras-Balderas, S., Domínguez-Cisneros, S. E. y Gómez-González, A. E. (2013). Riqueza y diversidad de peces continentales.
En La biodiversidad en Chiapas: estudio de estado. México, D.F: Comisión Nacional para el Conocimiento y Uso de la Biodiversidad/Gobierno del Estado de Chiapas.

Weber, A. y Wilkens, H. (1998). Rhamdia macuspanensis: a new species of troglobitic pimelodid catfish (Siluriformes: Pimelodidae) from a cave in Tabasco, México. Copeia, 4, 998-1004.

Weber, A., Allegrucci, G. y Sbordoni, V. (2003). Rhamdia laluchensis, a new species of troglobitic catfish (Siluriformes: Pimelodidae) from Chiapas, Mexico. Ichthyological Exploration of Freshwaters, 14, 273-280. 